\title{
L'école de la IIIe république et l'occitan
}

\section{Philippe Martel}

\section{OpenEdition}

\section{Journals}

\section{Édition électronique}

URL : http://journals.openedition.org/trema/1872

DOI : 10.4000/trema.1872

ISSN : 2107-0997

\section{Éditeur}

Faculté d'Éducation de l'université de Montpellier

\section{Édition imprimée}

Date de publication : 1 décembre 1997

Pagination : 101-115

ISSN : 1167-315X

\section{Référence électronique}

Philippe Martel, «L'école de la IIle république et l'occitan », Tréma [En ligne], 12-13 | 1997, mis en ligne

le 01 décembre 1997, consulté le 10 décembre 2020. URL : http://journals.openedition.org/trema/

1872 ; DOI : https://doi.org/10.4000/trema.1872

Ce document a été généré automatiquement le 10 décembre 2020.

Trema 


\title{
L'école de la IIIe république et l'occitan
}

\author{
Philippe Martel
}

1 Lorsqu'elle se met en place l'école de Jules Ferry (complétant un dispositif qui avait commencé à fonctionner bien avant) rencontre dans un certain nombre de régions, et notamment en Languedoc, un problème considérable: bien des enfants, en entrant à l'école, ignorent le français. Comment l'école primaire réagit-elle face à ce défi? Les réponses des historiens ont été variables. En laissant de côté ceux qui ont omis de se poser la question, on pourrait en gros distinguer deux familles de pensée. Il y a ceux qui considèrent que l'école a tout misé sur la répression, le refus absolu de prendre en compte la réalité des pratiques linguistiques des enfants. C'est le point de vue longtemps défendu par les occitanistes, par exemple ${ }^{1}$. D'autres, plus récemment, ont proposé une vision beaucoup plus nuancée. Tout en reconnaissant - non sans réticence - la réalité de pratiques parfois bien répressives, ils ont voulu montrer que, somme toute, les instituteurs, proches de leurs élèves par leurs origines géographiques et sociales, ont souvent été plus tolérants qu'on ne le croit vis-à-vis des "patois »- comme on dit en France. C'est la position développée dans la belle thèse de Jean-François Chanet, L'école de la République et les petites patries ${ }^{2}$. Pierre Boutan, s'appuyant sur les idées du linguiste Michel Bréal en 1872, nous paraît assez proche de cette position, même s'il signale les réactions mitigées de certains instituteurs de l'époque ${ }^{3}$.

2 C'est sur cette question que nous voudrions revenir ici, à partir de recherches que nous avons pu mener antérieurement ${ }^{4}$, et d'autre part, sur la foi d'un sondage, malheureusement trop rapide, à travers les revues consacrées à l'enseignement primaire et à ses maîtres dans le département de l'Hérault sous la III République. En essayant de voir ce qu'est la position « officielle » - si tant est, on le verra qu'il en existe vraiment une - et les réactions, sur le terrain, des praticiens que sont les instituteurs. En nous demandant s'il existe des textes proscrivant explicitement l'occitan - ou au contraire le valorisant - et en cherchant à déterminer l'attitude - les attitudes - des acteurs pédagogiques face à la langue d'oc. 


\section{Théories et débats}

3 Une première constatation s'impose : les textes officiels sont pratiquement muets sur la question de la langue. Elle n'apparaît que dans le règlement intérieur type proposé par le Ministère en 1881, et adopté ensuite par les départements ${ }^{5}$. On trouve là un article 14 ainsi libellé : «le français est seul en usage dans l'école ». C'est l'article le plus court de tout le texte. Et si Jules Ferry a éprouvé le besoin de gloser certains autres éléments de son règlement, celui-là ne pose visiblement aucun problème. D'autant moins, pourrait-on dire, qu'il ne fait que reprendre une disposition déjà présente dans la loi Falloux, et, audelà, la célèbre formule de l'ordonnance de Villers-Cotterêts qui, en 1539, imposait l'enregistrement des procédures judiciaires «en langaige maternel françois et non autrement ». Nulle part, les langues alternatives ainsi écartées ne sont nommées, fût-ce sous l'appellation englobante et péjorative de "patois ». D'entrée de jeu le règlement installe l'évidence d'une école monolingue par nature. Les textes ultérieurs ne seront pas plus explicites. Une circulaire sur les écoles maternelles de 1883 se borne à donner comme consigne aux maitresses : «corriger les défauts de prononciation ou d'accent local » des enfants $^{6}$. Ensuite, il faut attendre 1925 pour voir le ministre Anatole de Monzie signer une circulaire excluant explicitement les "patois» de l'école primaire - avec une argumentation assez précise quoique souvent spécieuse. Cette circulaire a eu un grand retentissement dans la presse régionaliste du temps. Mais elle est absente du Bulletin de l'Instruction Primaire de l'Hérault... Lacune? Oubli? Ou découverte rapide, par les bureaux compétents, du danger qu'il y avait à trop entrer dans les détails sur cette question?

4 Ceci dit, il faut croire que même sans texte spécifique l'école savait ce qu'elle avait à faire : le 23 novembre 1951 une circulaire ministérielle précise les modalités d'application de la Loi Deixonne votée presque un an plus tôt. Elle promet des «instructions pédagogiques » destinées à aider les maitres à utiliser « les parlers locaux » pour « l'étude de la langue française » (instructions qui, à notre connaissance, ne sont d'ailleurs jamais venues). Et surtout, elle se clôt sur cette phrase : «d'ores et déjà, l'interdiction absolue de l'utilisation éventuelle du dialecte, par exemple pour faire comprendre plus facilement des termes français abstraits, est levée $»^{7}$. Ce qui veut bel et bien dire qu'auparavant - et donc sous la III ${ }^{\mathrm{e}}$ République, cette «interdiction absolue » existait, même si aucun texte n'est cité en référence.

$5 \quad$ Autant dire que la question des langues distinctes du français n'apparaît qu'en creux dans les textes officiels, et que pour l'essentiel le législateur, dans sa sagesse, laisse toute latitude aux exécutants, sur le terrain, pour adopter d'eux-mêmes la bonne attitude.

6 Si nous abandonnons le domaine aride des lois et règlements pour chercher ailleurs des prises de positions officieuses des décideurs de la République, il faut nous rabattre sur les procès-verbaux des séances de la Chambre des Députés, où la question émerge de façon assez irrégulière, suivant un scénario bien rodé : un député (breton, souvent) pose une question orale au Ministre de l'Instruction primaire pour appeler son attention sur les « langues régionales ». Le Ministre répond, en substance, que la question est intéressante, et l'interpelle personnellement (car qu'il s'agisse de Leygues en 1902, de Doumergue en 1910 ou de Daladier en 1926, il est souvent lui-même occitan et occitano-phone) mais ne lui paraît pas devoir être traitée plus avant. Car l'école est là d'abord pour enseigner le français ${ }^{8}$. Moyennant quoi, le débat s'arrête avant d'avoir commencé. 
$7 \quad$ N'y a-t-il donc ici qu'à enregistrer un grand silence ponctué de quelques marmonnements ministériels ? La question qui nous occupe finit quand même par apparaître quelque part. Mais c'est dans l'univers officieux des publications pédagogiques nationales.

Il n'est pas question ici d'entrer dans le détail des débats menés dans ces publications9. Nous nous bornerons à résumer les positions qu'on y rencontre.

Il y a d'abord les partisans du tout-répressif, ceux qui utilisent la bonne vieille méthode empruntée à l'enseignement catholique, d'ailleurs - dite du «signal»: on la trouve exposée tranquillement par un inspecteur des Basses-Alpes du nom de Boitiat, dans la Correspondance Générale de l'Instruction publique du 13 septembre 1893 :

"Le matin, en entrant en classe, le maître remet au premier élève de la division supérieure un sou marqué d'une croix au couteau, ou de tout autre signe permettant de le reconnaître. Ce sou s'appelle le signe. Il s'agit pour le possesseur du signe, pour le signeur, comme disent les élèves, de se débarrasser du sou en le donnant à un autre élève qu'il aura surpris prononçant seulement un mot de patois."

Et à la fin de la journée, le maître n'a plus qu'à fondre sur le dernier malheureux possesseur du signe, pour le punir comme il se doit. Boitiat décrit avec une belle satisfaction les effets de ce système : les enfants se promènent en permanence avec un dictionnaire, qui permet à chacun de pouvoir " prouver péremptoirement au camarade qui l'accusera de parler patois que les mots qu'a prononcés ce camarade ne sont en effet nullement français ». Il évoque enfin cette petite fille qui n'ose plus parler, même chez elle, de peur qu'un de ces bons camarades ne soit caché derrière la fenêtre, prêt à lui infliger le "signe ».

Cette méthode, dont on est libre de penser tout ce que l'on veut, a effectivement été employée dans plus d'une école - sur ce point les témoignages sont nombreux, précis et concordants. Elle a bien sûr révulsé les félibres comme les militants du breton ou du basque, et elle a largement contribué à la mise en place de l'image négative de l'école primaire meurtrière des " patois » - et peut-être aussi d'autres choses - car ce n'est pas impunément qu'on s'exerce dès l'enfance à la délation. Ceci dit, on aurait tort de croire que l'attitude des pédagogues se limite à ce seul « innocent stratagème », pour reprendre la terminologie de Boitiat lui-même. En effet, la Correspondance publie dès ses numéros suivants des réactions pour le moins critiques d'inspecteurs ou d'instituteurs qui rejettent cette méthode. Les uns parce qu'ils estiment que la répression ne peut qu'endurcir les résistances. Les autres parce qu'ils considèrent, comme l'instituteur breton Hélie, qu' "exciter les enfants à espionner leurs camarades, à chercher des ruses pour les faire tomber dans un piège, les pousser à écouter aux portes est absolument mauvais"( Correspondance, octobre 1893, p. 378.)

Il y a donc d'autres attitudes, plus nuancées, comme par exemple la " méthode directe ", théorisée par Carré, un autre inspecteur exerçant ses talents en Bretagne, et développée par lui dans une revue spécialement destinée aux maîtres enseignant en zone non francophone, L'enseignement pratique. Cette méthode prétend se calquer sur la façon dont la mère apprend à parler à son enfant : elle lui parle normalement, sans employer le « babil » qui tient lieu de langage à l'enfant. A la longue, et à force d'imiter sa mère, celuici apprend «naturellement » à parler sa langue maternelle. Le maître, confronté à des enfants qui se sont visiblement trompés de langue maternelle, va donc leur enseigner la seule langue, la vraie, le français, en utilisant les mêmes procédés. En d'autres termes, il ne tiendra pas compte de ce qu'ils parlent en entrant à l'école, mais ne leur parlera que français. A la longue grâce à l'aide de tableaux illustrés, grâce aussi à l'infinie répétition 
patiente des mêmes mots par le maître, les enfants finiront par parler français. L'écrivain occitan Jean Boudou dans un roman en partie autobiographique, a décrit cette méthode, vue d'en bas. Il raconte comment, à son entrée à l'école, le maître l'a mis d'autorité à la « table des ânes ». "Là nous étions trois ou quatre petits monstres abandonnés à la grâce de Dieu. Nous pouvions gratter, dormir, croquer des échaudés, mais il ne fallait pas du tout parler, ou alors gare à la baguette... A force d'entendre les discours de Monsieur Peset, je finis par comprendre un peu de français. Quand il s'en fut aperçu, il me tira de la table des ânes pour me fourrer avec les savants qui avaient un alphabet $» .^{10}$

11 La «table des ânes »... Cette méthode est pourtant plus douce que celle du signal. Elle consiste tout simplement à faire comme si le " patois » n'existait pas, en laissant l'enfant régler lui-même la contradiction entre sa langue et celle de l'école. Et entre la culture de ses parents, dévalorisée - car ils n'ont pas su lui apprendre la «bonne » langue - et celle, étrange et incompréhensible au départ, des maitres. Ceci dit, tous les pédagogues ne sont pas convaincus de son excellence. Et la Revue pédagogique héberge en 1894 un grand débat sur le sujet, où s'affrontent un disciple de Carré et deux adversaires : un dénommé Perrin, qui rend compte de son expérience d'enseignement bilingue (mais c'est en Tunisie) et un certain Bobrovnikov, qui va dans le même sens, mais c'est à partir de son expérience d'inspecteur primaire en Sibérie, au pays des Tchouvaches et des Tchérémisses... Leurs arguments : à quoi bon faire comme si l'élève n'apportait rien avec lui en entrant à l'école, alors qu'il sait déjà parler autre chose qu'un simple babil ? Pourquoi ne pas utiliser ce qu'il sait - sa propre langue pour l'amener progressivement à découvrir la langue officielle?

Cette position, nuancée et pragmatique, a eu ses adeptes dans l'école française. Ils peuvent arguer du soutien d'un linguiste prestigieux, Michel Bréal, qui l'avait recommandée dans Quelques mots sur l'Instruction Publique, essai programmatique paru en 1872. Bréal recommande la comparaison entre le «patois » de l'élève et le français : ne s'agit-il pas de deux idiomes "frères ", même si l'un est mieux doté que l'autre ? Il admet même que le maître puisse lire aux enfants des textes d'auteurs du cru - des félibres par exemple - quand ces textes sont bons. L'enfant, argumente-t-il, n'en sera que plus fier de sa petite patrie, et cette fierté lui permettra de mieux aimer la grande patrie, la France. Une position du même type est défendue par exemple par l'instituteur ariégeois Beulaygue en 1897, dans L'enseignement du français à l'école rurale, ou par son compatriote l'inspecteur Combes, dans L'Enseignement Pratique du 18 Juin 1896, qui donne des exemples concrets : comment enseigner les mystères de l'accord du participe passé en se référant aux formes, non équivoques, de l'occitan ( «brassé/brassée = brassat/ brassado »). Là encore, des indices nombreux et concordants montrent que cette attitude était au moins aussi répandue que l'usage du signal.

13 Mais elle n'est pas sans poser quelques problèmes, pratiques et de principe. Il est clair que, pour Bréal comme pour ses disciples, les deux langues en présence ne sont pas égales. Il y a le "patois ", et il y a le français. Et il ne faut pas confondre. Beulaygue le dit sans détour, qui propose aux sceptiques d'essayer de traduire en "patois» un «passage d'un classique », et leur promet un intense embarras. Il s'agit bel et bien de convaincre l'enfant que seul le français est une vraie langue, quels que soient les services ponctuels que peut éventuellement rendre le patois. Par ailleurs, on devine que cette utilisation du «patois » ne peut fonctionner qu'avec une langue point trop éloignée du français dans ses structures. Du coup l'occitan, langue romane, est favorisé par rapport au breton, ou au basque. 
14 Et puis, il y a des limites. On laisse au maitre la possibilité d'utiliser le « patois » pour ses cours de langue française, mais on n'estime pas utile de lui enseigner comment l'utiliser au mieux. Pour cela il faudrait en effet commencer par lui apprendre à maîtriser les structures grammaticales du patois : il n'en est bien sûr pas question! Au début du siècle, on trouve sur le marché des petits guides, des "gasconnismes corrigés ", qui sur deux colonnes, établissent la comparaison terme à terme de l'occitan et du français et qui sont encore aujourd'hui précieux à qui veut, par exemple, travailler sur la syntaxe d'oc. Ces guides sont efficaces en ce qu'ils intègrent un savoir raisonné et une réflexion sur la langue qu'ils veulent détruire. Rien de tel à la fin du siècle. Au maître de se débrouiller. Or le français du maître, compte tenu de ses origines souvent populaires, est lui-même teinté d'occitanismes inconscients. N'étant pas dressé à les repérer dans ses propres pratiques, comment pourrait-il en protéger ses élèves? Nul doute que, dans bien des cas, la langue apprise par ces élèves a été moins le français que le francitan, ce français régional marqué par un fort substrat - phonologique, syntaxique et lexical - d'oc...

15 Enfin il y a une frontière à ne pas dépasser: le maître peut se servir, à des fins pédagogiques, du "patois », mais il n'est pas question qu'il l'enseigne. Le félibre Antonin Perbosc, instituteur du Tarn-et-Garonne, reçut un jour une note de son inspecteur ainsi libellée : "Je vous rappelle l'article $15 \mathrm{du}$ règlement, aux termes duquel le français est seul en usage à l'école. Je vous invite donc à ne plus donner des devoirs patois à vos élèves ». Perbosc avait commis une faute grave; il avait fait sortir le "patois» du seul domaine de l'oralité fugitive de sa salle de classe pour le laisser s'étaler indûment dans les cahiers de ses élèves. Or le domaine de l'écrit est tabou, réservé au seul français.

16 Cet inspecteur tarnais n'est d'ailleurs pas un Boitiat ; il cite même Bréal. Sa semonce n'en est donc que plus illustrative : le patois ne peut être que toléré, à condition qu'on n'en fasse pas trop étalage.

17 Ce n'est évidemment pas le point de vue défendu par Perbosc, et, au-delà de lui, par les félibres qui, dès les années 1870, commencent à revendiquer l'entrée de l'occitan à l'école primaire. Ils ne cesseront de le faire par la suite, sans se laisser rebuter par le peu d'écho qu'ils rencontrent du côté du ministère de l'Instruction Publique. ${ }^{11}$ Mais ils ne se contentent pas de revendiquer, ils font aussi des propositions. C'est ainsi que le frère Savinien met au point, aux débuts de la Troisième République, une méthode basée sur l'utilisation du provençal - à travers un système de thèmes et versions - pour l'apprentissage du français. Mais cette méthode, expérimentée par lui-même dans des écoles catholiques de la région avignonnaise, ne sortira pas de ce cadre. Même si la Correspondance Générale de l'Instruction Primaire la cite élogieusement en 1894...

D'une manière générale toutefois, l'école laïque et ses défenseurs manifestent une grande méfiance face à tout ce qui pourrait ressembler à une institutionnalisation de la présence de l'occitan dans les classes. C'est ce qui ressort des propos de l'Inspecteur Arnoux, qui, dans le cadre d'un ouvrage intitulé Les Troubadours et les Félibres du Midi (1888) consacre un chapitre (" Du patois dans les écoles») à l'éreintement des projets félibréens. Pour lui, la place des études patoises est à l'Université, pas à l'école primaire : on y a déjà assez de mal à enseigner le français ! Et puis, ne courrait-on pas le risque de voir l'Italie revendiquer la Provence, au nom de la similitude des langues? Plus tard, la Revue de l'enseignement Primaire du 2 avril 1896 publie un article d'un professeur d'École Normale du nom (basque d'ailleurs) de Bidart, repris d'une « conférence populaire » consacrée à la Provence. On trouve là un certain nombre de considérations ethnotypiques assez ordinaires à l'époque: "Le soleil dispose à la gaieté et à toutes les qualités qui en naissent: 
bonhomie, bonté facile, vie en plein air, donc sociabilité. La beauté du paysage inspire le culte des arts, la transparence de l'air grandit toutes choses et pousse à l'exagération »... On y trouve aussi une mention de la toute récente renaissance de l'occitan: "des poètes de génie (Mistral, Aubanel) ont écrit en provençal des œuvres aussi belles que les plus belles des œuvres françaises ». Ce n'est pas rien. Mais l'auteur ne va pas plus loin:

"Ce n'est pas une raison pour vouloir désapprendre le français. Dernièrement certains provençaux demandaient au ministre de l'Instruction Publique l'autorisation d'enseigner en provençal dans les écoles. Gardons nous de tomber dans cet excès de chauvinisme local. Honorons sans doute la langue de notre petite patrie, mais honorons aussi et aimons la langue de la grande patrie, de tous les français, le français ».

Voilà qui est clair. On a le droit d'aimer le patois. On peut même l'utiliser ponctuellement dans l'apprentissage du français. Mais il n'a pas à revendiquer une plus grande place à l'école.

C'est ainsi que l'on voit les choses, d'en haut. Il nous faut voir à présent ce qui peut se passer sur le terrain.

\section{Vu d'en bas...}

Nous avons sondé un certain nombre de publications spécialisées du Département de l'Hérault pour notre période. Et d'abord le très officiel Bulletin de l'Instruction Primaire du Département, à partir de 1876. Les références au problème qui nous occupe y sont rarissimes. Sur plusieurs dizaines de conférences pédagogiques portant sur les sujets les plus divers, nous n'en avons trouvé qu'une, organisée à Montagnac en 1900, portant explicitement sur "l'influence du patois dans l'enseignement de la langue française ». Le Bulletin n'en donne évidemment pas le contenu. ${ }^{12}$ Les rapports réguliers de l'Inspecteur d'Académie (à partir de 1900) parlent de tout, sauf du patois. Tout au plus l'inspecteur note-t-il que «le point faible, c'est la composition française "(Bulletin... $\left.\mathrm{N}^{\circ} 6,1904\right)$. Mais à la limite, est-ce bien spécifique aux seuls départements occitanophones? Sa seule concession à la prise en compte de la culture du lieu, c'est une notation ethnotypique dans un discours de distribution des prix des Écoles primaires supérieures, cette même année 1904. Il y annonce - de façon d'ailleurs mensongère - que son discours sera dépourvu de toute fioriture rhétorique, de "cette musique des mots dont nos oreilles méridionales peuvent, il est vrai, se montrer friandes, mais qui ne laisse après elle que le souvenir d'un bruit confus et vain ". Avant de récidiver en conclusion, pour inviter les maîtres à persévérer dans leur foi, leur zèle, et leur « méridionale bonne humeur ».

Il faut attendre le rapport de son successeur, en 1929, pour voir vraiment surgir la question de la langue. Cette année-là, l'Inspecteur glose sur l'attention qu'il faut porter au langage des élèves. Il le fait en termes séduisants :

«Il faut laisser les enfants s'exprimer à leur guise et dans leur langage, accepter leur vision $d u$ monde. Sans doute ils s'exprimeront à leur manière, qui n'est pas celle d'un auteur ni d'un pédagogue. Mais souvent leur langage parlé est plein de tournures vives et gracieuses qui ne sont pas nécessairement incorrectes [...] Il faut donc partir de la seule expérience réelle pour eux: le langage parlé».

Fort bien. Mais tout de suite après vient ce correctif: "évidemment ces remarques ne s'appliquent pas aux enfants qui ne parlent que le patois en dehors de la classe »13. Évidemment... Mais alors, si se pose vraiment le problème de ces enfants qui ne parlent pas le français 
chez eux, pourquoi en parler si peu? Nos pédagogues font comme si le problème ne se posait plus. Mais il leur arrive de laisser échapper, au détour d'une phrase, l'aveu qu'il se pose encore bel et bien... Et du côté des maîtres eux-mêmes? Nous avons consulté quelques revues professionnelles, en général plus syndicales que vraiment pédagogiques. Il s'agit du Midi scolaire et l'École du jeudi (réunis), qui a paru entre 1927 et 1929, de l'Hérault Enseignant, organe du Syndicat National des Instituteurs, entre 1922 et 1926, de la Forge, bulletin d'un groupe de jeunes instituteurs visiblement très proches du P.C.F. (1935-1937) et du Bulletin de l'Association Professionnelle des Instituteurs et Institutrices publics de l'Hérault (1920-1924), qui exprime les opinions de maîtres hostiles à la C.G.T. Pour l'essentiel, le contenu de ces revues est de nature strictement professionnel - il y est beaucoup question de statuts, de rémunérations, d'affectations, et de rapports avec l'Inspection. Il est beaucoup plus rare d'y rencontrer des articles pédagogiques. Par contre, elles ne dédaignent pas, à l'occasion, de se doter d'une rubrique culturelle, voire de s'ouvrir aux productions littéraires de leurs abonnés.

Si nous lisons ce corpus en fonction de notre sujet, nous constatons vite sa quasi absence de renvois à la situation linguistique régionale. L'Hérault Enseignant peutbien faire une place, dans un article pédagogique consacré à Séte, à l'ethnotype méridional: on y apprend en effet que le sétois est "insouciant, hâbleur, généreux, volontiers frondeur ", caractère qu'il doit au "mélange de races» qui a donné la population de la ville $\left(\mathrm{N}^{\circ} 57\right.$, octobre 1925), les références sont rares. Toutefois, dans sa partie plus proprement syndicale, il y a des choses à glaner dans le compte-rendu en octobre 1926, du Congrès de Strasbourg. Glay, secrétaire-adjoint du syndicat, y stigmatise, à propos de l'Alsace, l'aspiration à un "régime malsain de régionalisme", avant d'ajouter "il y a des basques, des bretons, des alsaciens, c'est entendu, mais il y a, par dessus toutes les particularités, la France ». On reconnaît là une méfiance à l'égard des cultures, ou des revendications « régionales », qui marquera d'ailleurs toute l'histoire du S.N.I., y compris au moment du vote de la loi Deixonne. Mais le Bulletin de l'Association professionnelle... qui ne cesse de polémiquer avec le Syndicat National, est tout aussi peu ouvert à la prise en compte des spécificités régionales. Le numéro d'avril 1920 offre ainsi une "lettre d'Alsace», danslaquelle l'Alsacien est décrit comme éminemment français : «vif, exubérant, tout proche de son frère méridional ». Tout au plus possède-t-il aussi la capacité de réflexion propre aux races du Nord et dont le « frère méridional » semble donc a priori dépourvu !

Et pourtant, ces revues font une place, parfois, à la différence" régionale. Mais exclusivement dans leurs rubriques culturelles. E. Malavialle, dans l'Hérault Enseignant peut rendre compte de romans "régionalistes": il célèbre les louanges de Pergaud, " conteur terrien », promis d'ailleurs à une belle fortune scolaire. De la fin prématurée de cet auteur franc-comtois, il tire un enseignement moral, bien dans l'air du temps : «loin de sa province, l'écrivain s'anémie " ( $\mathrm{N}^{\circ} 70$, novembre 1926). Le même, dans le $\mathrm{N}^{\circ} 67$, de juillet 1926, salue le régionaliste languedocien Georges Beaume, qui « reflète dans ses livres sa race et son pays ".

Mais il s'agit là d'auteurs en langue française. Et l'occitan? Aussi étonnant que cela puisse paraître, il est présent. Parfois sous la forme de citations : l'article sur Sète déjà signalé, et qui se poursuit sur plusieurs numéros, donne ainsi un refrain carnavalesque typique: "Maridàs, tenès-vous ben / Aqui i a la jouinessa qu'arriba " ( $N^{\circ} 54$, juillet 1925). Mais parfois aussi, l'occitan apparaît comme langue de création littéraire, dans les rubriques ouvertes aux œuvres des adhérents. Dans le même numéro, l'Hérault Enseignant publie un poème 
occitan (et en graphie "classique») de "notre sympathique camarade Arnaud»: il s'agit d'Albert Arnaud, dit « lou Pinsard », félibre depuis 1912 et lauréat du concours occitan de l'Académie des Jeux Floraux de Toulouse. Rien de particulièrement régionaliste, à la Pergaud, dans ce poème qui se veut regard nostalgique sur le parcours d'un couple, au soir de sa vie. Et on pourrait s'étonner de voir ici saluée une Académie toulousaine qui n'avait certes pas grand chose de commun avec les choix idéologiques affichés par le Syndicat! Mais puisqu'il s'agit de signaler la distinction flatteuse accordée à un « sympathique camarade »... Un autre camarade paré sans doute de qualités équivalentes, Avinens, donne lui aussi, de loin en loin, quelques petits textes familiers en occitan. Lui n'est pas félibre, signe que le goût de la langue pouvait dépasser le seul cercle des convaincus. Sur ce terrain, les rivaux ne sont pas en reste. Le Bulletin de l'Association professionnelle... publie en juin 1922, dans sa rubrique "le coin des poètes", un texte de l'instituteur cantalien (et félibre, et par ailleurs vieux syndicaliste) Louis Delhostal, «l'Institutour »... « traduit » en parler de Béziers par René Fournier, hôte régulier de la revue, instituteur lui-même, et accessoirement majorai du Félibrige ${ }^{14}$. Il reparaît d'ailleurs dès décembre 1922, cette fois-ci avec une de ses propres œuvres tandis qu'en décembre 1924, c'est tout bonnement l'animateur principal de la revue, Marcel Lignières, qui délaisse la polémique avec les mal-pensants pour saluer d'un poème occitan son vieux professeur d'École Normale, Paul Chassary, lui-même majorai du Félibrige. Marcel Lignières a été félibre luimême, du moins à certains moments. Son intérêt pour sa région l'a même amené à s'aventurer, bien plus tard, sur le terrain de l'histoire, avec un ouvrage consacré à la Croisade contre les Albigeois... ${ }^{15}$ Il n'est pas jusqu'à la Forge, l'organe "des jeunes de l'enseignement du Midi", qui ne fasse parfois une place à l'occitan, entre deux articles sur la condition féminine, ou la défense de l'U.R.S.S. Le numéro 21, de février 1937, publie ainsi un conte traditionnel, en occitan, concernant (et ridiculisant) les habitants de Nevian.

En bref, pour le monde des instituteurs, l'occitan n'est pas totalement une langue inconnue, ou uniformément détestée. Le prouve d'ailleurs la présence, dans le Félibrige, d'un certain nombre d'instituteurs, parfois à des postes importants. En 1914, parmi les majoraux du Félibrige, cinq sont ou ont été liés à l'Instruction primaire, soit $10 \%$ de l'effectif - à égalité, et ce n'est sans doute pas un hasard, avec les prêtres. Certains de ces majoraux - Perbosc, Estieu - ont joué un rôle considérable dans l'histoire de l'association. L'Hérault à lui seul fournit deux noms: Fournier, déjà entrevu, Gustave Thérond, directeur d'école à Sète. On peut y ajouter Paul Chassary, professeur à l'École Normale de Montpellier. A l'échelle de la totalité des effectifs félibréens - en tenant compte des adhérents de base, les «manteneire» - les instituteurs représentent $4 \%$ parmi lesquels huit héraultais. En 1936 encore, on retrouve la même proportion de maîtres d'école, parmi lesquels Arnaud et Lignières, nos vieilles connaissances. Et il n'y a pas de raison de considérer ces instituteurs comme totalement coupés des sensibilités politiques de leur corporation. Ce peut être en partie vrai pour Lignières ou Fournier, aussi peu à gauche que possible. Ce ne l'est assurément pas pour Gustave Thérond, militant de la S.F.I.O. avant 1914, et militant du PCF. après ${ }^{16}$. Le " patois » est même suffisamment légitime aux yeux de la corporation, à sa manière, pour qu'on le voie parfois affleurer au cœur même de l'institution que représente l'École Normale. Nous renvoyons ici à ce que dit JeanFrançois Chanet des débuts de l'auvergnat Louis Delhostal, ou de l'organisation de cours de provençal dans l'École Normale du Var entre les deux guerres, ou de la participation des instituteurs béarnais aux concours organisés par l'escole Gastou Fébus, ou de la 
présence de l'occitan dans tel manuel de lecture départemental ${ }^{17}$. On pourrait y ajouter l'action de certains inspecteurs animateurs de revues pédagogiques, comme le félibre Anfos Martin et sa revue le Bassin du Rhône à la veille de la guerre de 1914. On pourrait enfin signaler que la Ligue pour la Langue d'Oc, à l'École de Jean Bonnafous, fondée dans les années 1920 pour relayer les revendications félibréennes en matière d'enseignement, compte dans ses rangs, dès le début, 1891 instituteurs publics. ${ }^{18} \mathrm{Il}$ suffit de chercher un peu pour voir surgir en foule les exemples.

Bien sûr, il ne faut rien exagérer. Rapporté au nombre total des instituteurs du département de l'Hérault, nos félibres doivent représenter quelque $0,4 \%$. Il est peu probable que les autres départements fassent beaucoup mieux. Tout au plus quelques maîtres d'école peuvent-ils adhérer, par ailleurs, à des groupes félibréens locaux, sans s'inscrire au Félibrige lui-même. Ceci dit, la proportion des instituteurs dans d'autres associations culturelles - on pense par exemple aux Sociétés savantes locales, au recrutement à vrai dire souvent élitiste - ne doit pas être bien plus forte.

Plus grave : nous savons que tel ou tel instituteur a pu être félibre, ou a pu taquiner la muse d'oc dans les colonnes de sa revue syndicale. Mais cela ne nous dit rien sur la façon dont il pouvait traiter le «patois » dans sa pratique quotidienne, au contact de petits paysans qui ne faisaient certes pas de beaux vers. Pour un Perbosc qui donne des « devoirs patois » à ses élèves, jusqu'à ce que son inspecteur le rappelle à l'ordre - ou qui les lance à la recherche des contes ou des proverbes de leur coin - combien y a t-il eu de maîtres qui ne mélangeaient pas les genres, et ne retrouvaient la langue d'oc que dans le secret de leurs heures de loisir?

Or c'est bien vers le second cas de figure que nous oriente la lecture de nos revues héraultaises. Rien sur le patois dans les articles sérieux, mais une petite place, sous le signe de la connivence et de la convivialité, dans le supplément « littéraire ». La langue d'oc ne serait-elle donc, ne saurait-elle être qu'un hobby, soigneusement distingué de la vie professionnelle ? Où l'on retrouve le dédoublement cher à Chanet, entre une sphère publique toute française, et une sphère privée, celle de la culture personnelle et du loisir, où l'occitan peut apparaître sans dommage.

33 Au total, nous éprouvons, au terme de cette enquête, une lancinante impression de flou. L'occitan est là, mais sans y être. Il apparaît en creux dans les instructions officielles, on le croise parfois, mais comme par hasard, dans la prose des pédagogues, on le voit surgir sans crier gare au moment où on s'y attend le moins, sous la forme de vers plus ou moins bien venus épars dans de minces bulletins. Le tableau qui se dégage de l'ensemble est contrasté. Il y a eu des instituteurs pour manier le signal, mais d'autres s'y sont refusés.

Il faut pourtant aller plus loin. On aura compris que les deux points de vue mentionnés au début de cet article - l'école a opprimé l'occitan / L'école n'a pas opprimé l'occitan - sont tous deux trop sommaires, et par ailleurs trop marqués d'arrière-pensées idéologiques, pour rendre compte de la réalité. Par contre, nous pouvons nous interroger sur le fait que, somme toute, ce problème a suscité bien peu d'interventions officielles ou officieuses, comme nous pouvons nous interroger sur le fait que si peu d'historiens s'y soient intéressés.

Nulle loi ne réprime l'usage de l'occitan, ou de toute autre langue. Peu de circulaires se penchent sur la question de la langue, à l'exception de la circulaire de Monzie, promptement enterrée. Il y a, bien sûr, des articles savants consacrés à l'enseignement du français langue étrangère dans nos revues pédagogiques. Mais sur la masse de papier que 
représentent ces revues, force est de constater que la question qui nous intéresse ne les mobilise que marginalement. Ce qui n'enlève rien à la qualité intrinsèque des études publiées, et des débats qui se mènent à travers eux. Mais précisément ces débats ne sont jamais tranchés. Bréalistes, savinianistes, carréistes, signalistes: tous font leur apparition, donnent leur point de vue, sans plus. Aux lecteurs de se faire leur opinion. Mais au fait, combien d'instituteurs de base ont-ils vraiment lu ce type de littérature?

Ce qui nous amène à une conclusion brutale, mais qui nous permet de mettre d'accord, en les renvoyant dos à dos, les deux avis extrêmes que nous citions plus haut: la question linguistique en France n'a absolument pas été pensée par l'école primaire.

Elle n'a pas été pensée pédagogiquement. Il n'y a eu ni proscription généralisée et explicite des "patois", ni capacité à mettre au point une méthode efficace pour les utiliser dans l'enseignement, ni préparation réelle pour des enseignants appelés à fonctionner en face de petits alloglottes. Ce qui règne, c'est le bricolage, plus ou moins brutal, plus ou moins heureux, rarement astucieux.

Elle n'a pas été pensée politiquement ni, dirions-nous, d'un point de vue civique. «Il y a des manières de répandre une langue, et c'est proprement un crime envers les citoyens que d'avoir systématiquement banni les parlers locaux de l'école primaire ». C'est Van Gennep qui tient ces rudes propos dans une mince brochure de $1911:$ La décadence et la persistance des patois. Mais Van Gennep, ethnologue et marginal, n'a rien à voir avec le monde de l'école. Pas plus que Jaurès, qui aborde la question la même année dans la Revue de l'enseignement primaire. S'il voit les avantages d'une éducation bilingue, nulle trace, chez ce responsable socialiste doublé d'un penseur, d'une réflexion sur le statut politique et social du problème linguistique en France. Nul ne semble s'être posé de questions sur le défi que représentait, pour la définition de la Nation française, la coexistence possible de plusieurs langues. Ni sur la contradiction qu'il pouvait y avoir entre une affirmation généreuse mais théorique de l'égalité de tous, et l'inégalité de fait que représentait la non-prise en compte des langues parlées par des millions de citoyens.

Elle n'a pas été pensée culturellement. Même les plus chauds partisans d'une prise en compte des " patois » n'entendent pas laisser ces «patois » jouer dans le même registre que la langue nationale, nonobstant les compliments machinaux adressés parfois aux Félibres. Il est clair que, pour tous ces gens, les « patois » ne sont pas des langues, et n'ont donc pas de vraie littérature. Même la présence de petits poèmes d'oc dans les colonnes de telle ou telle revue ne change rien au fond du problème car ils demeurent marginaux et minoritaires par rapport aux productions en français. En d'autres termes, et ceci rejoint notre point précédent, l'idée que la culture française puisse être plurielle et s'enrichir de cette pluralité est à cent lieues des esprits du temps. Seule la culture française est une vraie culture...

Reste à savoir pourquoi la question n'a pas été pensée. Plusieurs raisons peuvent l'expliquer.

41 Il ne s'agit pas d'un problème national. D'abord parce qu'il ne concerne pas l'ensemble du territoire et de la société françaises, mais seulement ses périphéries - et les moins développées de surcroît. Il n'y a donc pas lieu de légiférer, depuis le centre, sur une question qui ne le concerne pas. De plus, la question linguistique n'est pas un de ces problèmes de société qui émergent fatalement à travers un conflit, et qu'il faut donc bien se résoudre à régler. La société française se divise sur le statut de la religion par exemple, ou sur la propriété des moyens de production. Mais la diversité linguistique n'est pas, et 
ne sera jamais - sauf en Alsace, après 1918 - un lieu de clivage. Droite et gauche, cléricaux et libres penseurs ont sur ce point une attitude largement commune, se partageant entre adversaires ou partisans des patois sans que cela compte vraiment. Quant aux principaux intéressés, les locuteurs des langues autres que le français, leur désir d'acquérir le français est indéniable dès la fin du XIX ${ }^{\mathrm{e}}$ siècle, et dans bien des régions sans doute bien avant. Que l'Église, localement, ait parfois pu voir dans les langues minoritaires un rempart contre les idées nouvelles ne suffit pas à leur donner une place stratégique dans les débats du temps - d'autant moins que l'Église elle-même est souvent aussi francophone que le camp d'en face. Et, bien sûr, nul ne prend vraiment au sérieux l'éventualité de mouvements séparatistes se nourrissant, contre la France, des spécificités « régionales ». En balayant d'un revers de main les « patois » pour mieux faire avancer le français, l'École, et au delà l'Etat pouvait croire en toute sincérité ne faire que refléter un consensus tellement massif qu'il n'avait plus besoin d'être formulé.

Mais il y a autre chose, que l'on distingue à travers les propos des partisans de la méthode Carré sur le niveau culturel des petits patoisants. Ou qui se lit dans les plaintes régulières de nos revues syndicales à propos des "mauvais postes » ou des " postes déshérités ", ces écoles de hameau où le pauvre instituteur, la pauvre institutrice, sont au contact avec des populations grossières.

Ce qu'il y a là, c'est tout bonnement le mépris pour ce bas-peuple qu'on commence à peine à éduquer et qui a encore bien du chemin à parcourir. Un enfant, un adulte qui pense en " patois » ne pense pas, pour tous ces gens. Il est donc inutile de penser le statut de l'idiome dans lequel il pense. Quant à le valoriser comme élément de la culture nationale... nous sommes sur ce point bien moins indulgent que Jean-François Chanet, et sa théorie du dédoublement, en vertu de laquelle le "patois ", absent de l'école, aurait toute latitude de triompher au dehors, y compris dans l'oralité des maîtres eux-mêmes, en dehors de leurs heures de service. Il y aurait donc une langue publique, le français, et une langue privée, le patois, chacune dans sa sphère propre pour le plus grand bien des valeurs républicaines. C'est méconnaître le fait que les deux langues ne sont ni égales, ni également légitimes. Absent de l'école, cette porte de la promotion sociale, du moins en théorie, comment le " patois » pourrait-il bénéficier du moindre prestige, dans la société qui l'utilisait jusque là normalement? Il est perçu au mieux comme inutile dans le nouveau monde qui se met en place, au pire comme un obstacle à l'ascension de ses locuteurs qui, dès lors, n'auront de cesse de s'en débarrasser. La hiérarchie établie par le système entre les langues (et qui reflète, au demeurant, faut-il le rappeler ? la hiérarchie sociale, tout simplement) rend impossible leur cohabitation harmonieuse. Quant à la place, indéniable, de la référence au « pays » et à la région dans l'enseignement primaire, elle ne nous paraît pas contradictoire de ce qui précède. C'est une chose de montrer aux enfants ce qu'est un château fort en leur montrant les mines du château voisin, c'est une chose de célébrer à leur bénéfice les bienfaits de la vie à la campagne; c'en est une tout autre de laisser leur langue entrer dans l'école. Là, plus question de suivre l'adage « du connu à l'inconnu ». Célébration du pays natal et refus de la langue de ce pays peuvent tout à fait cohabiter - et cohabitent fort bien, à notre sens - parce qu'ils s'inscrivent dans un même projet implicite : la mise en conformité des mentalités et de la culture populaire avec les modèles «nationaux» dominants. La mode régionaliste, avec son contenu souvent moralisateur, est le versant doux de ce projet, la mise hors jeu du vécu linguistique des enfants est son versant dur. 
Le problème n'est donc pas pensé, puisqu'il n'a pas besoin de l'être. Ce qui ne l'empêche bien sûr pas de se poser.

On peut ainsi se demander si l'ignorance délibérée de l'occitan a vraiment permis aux enfants d'apprendre plus facilement le français. Quel français d'ailleurs? Un problème qui n'est jamais posé par nos textes, c'est celui du francitan, ce français régional résultant du placage des structures phonologiques, syntaxiques et lexicales de l'occitan sur le français de l'école. Ce francitan, qui n'est pas vraiment le français théorique de nos pédagogues, a survécu jusqu'à nos jours, y compris d'ailleurs dans les performances linguistiques des instituteurs eux-mêmes. Petite revanche du " patois » sur la langue des maîtres, mais revanche inévitable à partir du moment où nulle réflexion n'est menée sur les différences entre les deux langues. Nous avons évoqué plus haut ces « gasconnismes corrigés» qui fleurissent aux débuts du siècle pour disparaître avec la montée en puissance de l'école. Ils auraient pourtant été bien utiles. Sans eux, sans leur travail d'inventaire patient, quoique malveillant, des points sur lesquels l'occitan imprime sa marque, souterrainement, au français du Midi, que pouvaient faire nos instituteurs? Paradoxalement, le refus de prendre en compte la langue des enfants a permis le développement, à l'oral, d'un français bien différent de celui que souhaitaient voir triompher les défenseurs de la langue nationale unique.

Une dernière remarque, qui nous ramène à ces instituteurs obligés de se débrouiller, tant bien que mal, avec la réalité du contact des langues sur le terrain.

Ils ont joué, globalement, le rôle qu'on leur demandait de jouer. Nul doute que certains n'y aient mis un zèle particulier, décuplé par leur propre malaise face à cette langue de leur propre enfance qu'on leur suggérait de chasser de l'enfance de ceux dont ils avaient la charge. Parmi les utilisateurs du «signe», combien d'ex- "patoisants» qui ne se pardonnaient pas de l'avoir été et réglaient, à travers la répression, leurs propres comptes avec leurs origines? Mais, contradictoirement, comment ne pas penser à ces maîtres pour qui la langue d'oc, exclue du système, n'en continuait pas moins à exister, et qui, une fois posée la blouse grise de leur sacerdoce, $\mathrm{y}$ revenaient, à travers ces poèmes plus ou moins malhabiles qu'ils envoyaient ensuite aux revues que lisaient leurs collègues? Sous l'apparente efficacité du monolinguisme d'État, combien de souffrances intimes, chez les enfants comme chez les instituteurs?

\section{NOTES}

1. Voir, le Petit livre de l'Occitanie, Ardouane, Comité Occitan d'Études et d'Action, 1971, qui titre : "l'école du mépris et de l'ignorance ». Ou, de Claude GENDRE et Françoise JAVELLIER, École, Histoire de France et minorités nationales,Lyon, Fédérop, 1978.

2. CHANET J.-F., L'école de la République et les petites patries,Paris, Aubier, 1996.

3. BOUTAN P., "Michel Bréal, ami des patois : linguistique, pédagogie, politique ", in Langages, décembre 1995, pp. 33-51.

4. Nous avons abordé ce problème en passant, dans notre thèse d'État, sous la direction de Maurice AGULHON Les Félibres et leur temps : renaissance d'oc et opinion, 1850-1914,Paris I, 1993. Et 
dans un article de la revue pédagogique Lenga et Païs d'oc, $\mathrm{N}^{\circ} 23,1991$ : «Les pédagogues et les patoissous la III République », pp. 11-22).

5. Bulletin de l'Instruction Primaire, Hérault, $1880, \mathrm{~N}^{\circ}$ 9, p. 137. texte «modèle » et $1881, \mathrm{~N}^{\circ} 4$ règlement départemental.

6. Ibid.,1883, $\mathrm{N}^{\circ} 1$, p. 13.

7. Circulaire citée dans un document du C.R.D.P. de l'Académie d'Aix-en-Provence, Langues et cultures régionales,datéde janvier 1968. A cette date, c'est, avec la Loi Deixonne, elle aussi reproduite dans le document, l'essentiel de la littérature réglementaire et législative française sur la question...

8. Cf.Philippe Martel: «Le seuil de tolérance: les hommes politiques et l'occitan sous la troisième République", in Les Français et leurs langues,Dir. Jean-Claude BOUVIER et Claude MARTEL, Aix-en-Provence, 1991, pp. 259-273.

9. Voir, pour plus de détails, le livre de Jean-François CHANET, et notre propre article cité plus haut sur les pédagogues et les patois.

10. La Grava sul camin,Toulouse, I.E.O., 1956, pp. 96-98. Traduction française de l'auteur.

11. Cf.notre article "L'impossible politique linguistique occitaniste », in Lengas $\mathrm{N}^{\circ} 25,1989$, pp. 51-70. A noter qu'aux tous débuts - dans son discours d'Apt en 1862, par exemple - Mistral milite contre l'école primaire, susceptible selon lui de couper les enfants de leur culture d'origine. Il lui faut un certain temps pour comprendre que le processus de scolarisation des enfants des campagnes est irréversible, et qu'il faut essayer de l'accompagner.

12. Bulletin... $\mathrm{N}^{\circ} 2$, février/mars 1900.

13. Bulletin... 1929, $\mathrm{N}^{\circ} 6$, p. 208.

14. Rappelons que les Majoraux, au nombre de cinquante et cooptés à vie, représentent en quelque sorte l'état-major du Félibrige.

15. L'Hérésie Albigeoise et la Croisade,Paris, 1964. Fidèle à ses engagements de jeunesse, Lignières y manifeste un grand amour de l'ordre social menacé par l'hérésie et un patriotisme français dépourvu de tout état d'âme.

16. Chiffres et noms tirés des Cartabèu de Santo Estèllode 1914 et 1936. Le Cartabèu est en quelque sorte l'annuaire des Félibres.

17. CHANET, op cit., passim,et notamment pp. 134 sqq., 338 sqq.

18. Revue Oc, Toulouse, $\mathrm{N}^{\circ}$ 2, février 1924. Sur ce nombre: 5 Héraultais, 2 Gardois, 6 Audois, 1 Lozérien. Les départements qui fournissent le plus d'instituteurs membres de la Ligue sont les Basses-Pyrénées (13), le Var (12) et la Dordogne (10).

\section{RÉSUMÉS}

Non disponible

Not available

\section{INDEX}

Keywords : French, language, occitan language, primary school, third republic

Mots-clés : école primaire, français, IIIe république, Languedoc, occitan 
AUTEUR

PHILIPPE MARTEL

CNRS, Université Paul Valéry de Montpellier 